\title{
EDITORIAL
}

\section{Opioids for Chronic Noncancer Pain: As the Pendulum Swings, Who Should Set Prescribing Standards for Primary Care?}

\author{
Michael R. Von Korff, ScD \\ Group Health Research Institute, Seattle, Washington
}

Ann Fam Med 2012;10:302-303. doi:10.1370/afm.1422.

$\mathrm{P}$ rimary care is the vital center of compassionate, evidence-based, and safe management of chronic pain. It is where long-term opioid prescribing for chronic noncancer pain has most often occurred, and it is a gateway to nonpharmacologic approaches to chronic pain care.

Rosenblatt and Catlin offer compelling reasons for more selective and cautious opioid prescribing for chronic noncancer pain in primary care. ${ }^{1}$ The role of opioids in management of chronic noncancer pain is a timely and consequential clinical issue currently being considered by the Food and Drug Administration (FDA) as a result of epidemic levels of drug overdose and addiction involving prescription opioids. On May 30-31, 2012, the FDA's Center for Drug Evaluation and Research convened a public meeting on the campus of the National Institutes of Health to consider use of analgesics in management of chronic noncancer pain, focusing on prescription opioids. The purpose of the meeting was to inform the FDA's regulatory responsibilities regarding analgesic medications, to review what is known, and to identify gaps in knowledge regarding the effectiveness of analgesics in the management of chronic noncancer pain. I attended this meeting as a participant and presenter. As a researcher who has studied the management of chronic pain in primary

Conflicts of interest: author reports none.

\section{CORRESPONDING AUTHOR}

Michael R. Von Korff, ScD

Senior Investigator

Group Health Research Institute

1730 Minor Ave, Suite 1600

Seattle, WA 98101

vonkorff.m@ghc.org care settings for more than 2 decades, I offer a brief overview distilling information relevant to issues facing primary care physicians in their efforts to provide safe, evidence-based, and compassionate management of chronic pain.

FDA officials set the context for the meeting, stating that epidemic levels of prescription opioid abuse and overdose are major public health concerns. In a public hearing, patients, family members, and clinicians also shared their perspectives. Despite diverse views and backgrounds, the meeting provided a balanced and clear overview of what is known-and what needs to be learned-about the effectiveness and safety of opioids for chronic noncancer pain.

Neuroscientists, clinical pain researchers, and epidemiologists presented recent findings on chronic noncancer pain and opioids. Srinvasa Raja of the Johns Hopkins School of Medicine reviewed evidence from randomized controlled trials. He reached conclusions similar to Papaleontiou et al, whose meta-analysis found that "short-term use of opioids (for chronic pain) is associated with modest but favorable effects on pain and physical function." Jane Ballantyne of the University of Washington School of Medicine, reviewing evidence from observational studies, suggested that results of short-term trials may not generalize to longterm opioid use or to patient populations less carefully selected than those enrolled in trials. Uncertainty about the long-term effectiveness of opioids for chronic noncancer pain was identified as a major gap in knowledge.

Several speakers noted important individual differences in clinical response to long-term use of opioids for chronic noncancer pain. Inadequate understanding of who achieves clinically meaningful benefits from long-term opioid use and who does not was identified as a second major gap in knowledge. As risks are inher- 
ent in long-term opioid use, and benefits are uncertain, Ed Covington of the Cleveland Clinic stated that initiation of opioids for management of chronic pain should always be presented to patients as a trial, and that opioids should be discontinued early whenever clinically meaningful improvements in pain and participation in life activities are not achieved.

Conference participants observed that improved understanding of the long-term consequences of dose escalation was needed. Patients escalated to higher opioid dose-from which discontinuation is often difficult_-are at increased risk of adverse events, whereas the long-term efficacy of high-dose regimens for chronic noncancer pain has not been established Comparing the effectiveness and safety of nondrug treatments to pharmacologic management of chronic pain was also identified as an important research need. This meeting did not seek to define new practice standards; however, there was a collective recognition that new approaches are urgently needed to increase the effectiveness and safety of chronic pain management.

Although the appropriate role of opioids in longterm management of chronic noncancer pain remains unclear, practice standards for long-term opioid prescribing are likely to change for the second time in a generation. In the 1990s, palliative care specialists defined opioid prescribing standards for chronic noncancer pain. ${ }^{3}$ These guidelines, based almost entirely on expert opinion, were then generalized to primary care practice without research, evaluation, or meaningful input from primary care physicians. As the pendulum swings in the direction of more-selective and-conservative opioid prescribing, it is critical that primary care physicians take the leading role in defining how, when, and for whom opioids should be used in long-term management of chronic pain.

To read or post commentaries in response to this article, see it online at http://www.annfammed.org/content/10/4/302.

Key words: opioids; chronic pain

Submitted June 6, 2012, accepted June 6, 2012.

\section{References}

1. Rosenblatt RA, Catlin M. Opioids for chronic pain: first do no harm. Ann Fam Med. 2012;10(4)300-301.

2. Papaleontiou M, Henderson CR, Turner BJ, et al. Outcomes associated with opioid use in the treatment of chronic noncancer pain in older adults: a systematic review and meta-analysis. J Am Geriatr Soc. 2010;58(7):1353-1369.

3. American Pain Society. The use of opioids for the treatment of chronic pain. A consensus statement from the American Academy of Pain Medicine and the American Pain Society. Clin J Pain. 1997;13(1):6-8. 